\title{
Plantas com possível atividade hipolipidêmica: uma revisão bibliográfica de livros editados no Brasil entre 1998 e 2008
}

\author{
PIZZIOLO,V.R. ${ }^{1 *}$; BRASILEIRO, B.G. ${ }^{2}$;OLIVEIRA, T.T. ${ }^{1}$; NAGEM, T.J. ${ }^{3}$ \\ ${ }^{1}$ Departamento de Bioquímica e Biologia Molecular, ${ }^{2}$ Departamento de Fitotecnia, Universidade Federal de Viçosa - \\ UFV, Avenida PH Rolfs, s/n, CEP:36570-000, Viçosa-Brasil *virginia.pizziolo@ufv.br ${ }^{3}$ Universidade Federal de \\ Ouro Preto - UFOP, Departamento de Química, Campus Universitário, Morro do Cruzeiro, s/n, CEP: 35400-000, \\ Ouro Preto-Brasil
}

\begin{abstract}
RESUMO: O objetivo deste trabalho foi realizar levantamento sobre as plantas medicinais indicadas para o tratamento da hiperlipidemia, hipercolesterolemia e/ou aterosclerose. Este estudo foi feito a partir da pesquisa bibliográfica em 32 livros publicados no Brasil entre 1998 e 2008, resultando em lista de 85 espécies distribuídas em 53 famílias, principalmente Asteraceae e Fabaceae, com 54 espécies exóticas e 31 nativas. As espécies mais citadas foram Allium sativum (Alliaceae), Cynara scolymus (Asteraceae), Curcuma longa (Zingiberaceae), Allium cepa (Alliaceae), Echinodorus grandiflorus (Alismataceae), Taraxacum officinale (Asteraceae), Vernonia condensata (Asteraceae), Cuphea carthagenensis (Lythraceae) e Curcuma zedoaria (Zingiberaceae). As espécies nativas, citadas em pelo menos 2 livros, foram investigadas para alguma evidencia científica relacionada a possíveis efeitos hipolipidêmico, hipocolesterolêmico e/ou contra aterosclerose. Somente foram encontrados estudos científicos, relacionados a estes efeitos, para as espécies nativas Cuphea carthagenensis e Echinodorus grandiflorum, indicando a necessidade de pesquisas que possam garantir a segurança e a eficácia do uso destas espécies.
\end{abstract}

Palavras-chave: aterosclerose, hipercolesterolemia, hiperlipidemia, plantas medicinais

\begin{abstract}
Plants with possible hypolipidemic activity: a review of books published in Brazil between 1998 and 2008. The aim of this study was to survey medicinal plants indicated for the treatment of hyperlipidemia, hypercholesterolemia and/or atherosclerosis. This study was performed from a review of 32 books published in Brazil between 1998 and 2008, resulting in a list of 85 species belonging to 53 families, especially Asteraceae and Fabaceae, with 54 exotic and 31 native species. The species most often cited were Allium sativum (Alliaceae), Cynara scolymus (Asteraceae), Curcuma longa (Zingiberaceae), Allium cepa (Alliaceae), Echinodorus grandiflorus (Alismataceae), Taraxacum officinale (Asteraceae), Vernonia condensata (Asteraceae), Cuphea carthagenensis (Lythraceae) and Curcuma zedoaria (Zingiberaceae). Native species cited in at least 2 books were investigated for some scientific evidence related to possible hypolipidemic and hypocholesterolemic effects and/or action against atherosclerosis. Scientific studies related to these effects were found only for the native species Cuphea carthagenensis and Echinodorus grandiflorum, indicating the need for studies capable of assuring the safety and the efficacy as to the use of these species.
\end{abstract}

Key words: atherosclerosis, hypercholesterolemia, hyperlipidemia, medicinal plants

\section{INTRODUÇÃO}

Os últimos 30 anos tem presenciado um declínio razoável da mortalidade por causas cardiovasculares em países desenvolvidos, enquanto que elevações rápidas e substanciais tem ocorrido em países em desenvolvimento, dentre eles o Brasil. De acordo com a OMS, esta tendência de aumento na doença cardiovascular tende a persistir, agravando ainda mais o quadro de morbidade e mortalidade elevadas nos países em desenvolvimento. Caso medidas preventivas não sejam tomadas, prevê-se uma verdadeira epidemia de doença cardiovascular com consequências desastrosas para a saúde

Recebido para publicação em 10/06/2009

Aceito para publicação em 29/03/2010

Rev. Bras. PI. Med., Botucatu, v.13, n.1, p.98-109, 2011. 
pública (IV Diretrizes da SBC, 2007).

Dentre as doenças cardiovasculares, a aterosclerose se caracteriza pelo depósito de colesterol nas artérias com formação de placas gordurosas que obstruem a passagem do sangue (Cotran et al., 2000). Um dos fatores que contribuem para a aterosclerose é a alta concentração de lipídeos no sangue (hiperlipidemia), caracterizada pelo aumento do colesterol (hipercolesterolemia) e dos triglicerídeos (hipertrigliceridemia). Estes são transportados pelas lipoproteínas, como por exemplo, a lipoproteína de baixa densidade (LDL) cuja alta concentração está relacionada com o risco maior de desenvolvimento da aterosclerose e da doença coronária cardíaca (DCC). Sendo a aterosclerose uma doença multifatorial que avança sem sintomatologia aparente, a detecção de hiperlipidemia (hipercolesterolemia e hipertrigliceridemia) e a prevenção podem estar ligadas à diminuição do risco da doença aterosclerótica (IV Diretrizes da SBC, 2007).

Para a hipercolesterolemia e para aterosclerose, consequentemente, diversas formas de intervenções, preventivas, curativas ou de redução têm sido utilizadas. Intervenções medicamentosas mostram efeitos positivos, porém o custo elevado e os efeitos colaterais de diversas drogas têm despertado interesse de pesquisadores em conhecer os efeitos de substâncias naturais na redução dos níveis de colesterol (Moll, 2006).

$\mathrm{Na}$ última década, a busca de novos medicamentos para o tratamento da aterosclerose tem valorizado muito o papel de suplementos, alimentos alternativos e fitoterápicos que ajudem na redução de triglicerídeo e colesterol das lipoproteínas plasmáticas (Anila \& Vijayalakshmi, 2002; Borradaile et al., 2003; Whitman et al., 2005; Jung et al., 2006; Marinangeli et al., 2006; Brusq et al., 2006).

Produtos naturais derivados de plantas têm sido testados em animais e humanos com resultados positivos para os efeitos hipocolesterolêmicos (Jeon et al., 2004; Kong et al., 2004). Flavonóides isolados ou purificados de plantas agem inibindo enzimas da biossíntese e absorção do colesterol como a hidroximetil-glutaril CoA redutase (HMGCoA redutase), bem como enzimas lipogênicas (glicose 6 -fosfato desidrogenase e enzima málica) e do metabolismo lipídico como lipoproteína lipase e a lecitina colesterol acil transferase (LCAT). Estas foram dosadas em fígado, tecido adiposo e plasma de ratos alimentados com dieta contendo colesterol (Anila \& Vijayalakshmi, 2002; Jung et al., 2006). Modelos in vitro de vários tipos de cultura celular têm sido utilizados por grandes laboratórios de pesquisas e indústrias farmacêuticas para comprovar, a nível molecular, o mecanismo de ação pelos quais os flavonóides são eficazes em reduzir a concentração de colesterol do sangue (Borradaile et al., 2002; Whitman et al., 2005; Brusq et al., 2006).
A fitoterapia é modalidade de tratamento que vem crescendo nos últimos anos, principalmente em função do alto custo dos medicamentos industrializados (Yunes et al., 2001). Apesar de muito usada como alternativa para os tratamentos de doenças como câncer, aterosclerose, diabetes, hipertensão, dentre outras, pesquisas científicas devem ser feitas sobre a atividade farmacológica das plantas bem como os efeitos tóxicos (Singi et al., 2005).

As plantas representam importante fonte de drogas considerando a grande quantidade de moléculas com potencial medicinal, podendo contribuir efetivamente na busca de novos produtos bioativos. Há vários relatos de plantas e constituintes químicos com atividade hipoglicemiante, hipotensiva, hipocolesterolêmica, antiaterosclerótica e antitrombótica utilizadas na medicina chinesa (Wang \& Ng, 1999; Coon \& Ernst, 2003; Moll, 2006).

A necessidade de dispor de agentes ativos frente a alterações do perfil lipídico tem levado a pesquisa de produtos naturais que tenham efeito na redução do colesterol e lipídeos plasmáticos. A atividade hipolipemiante tem sido encontrada em várias espécies medicinais como a Camellia thea, Glycine max, Plantago sp., Garcinia cambogia e em outros constituintes vegetais como os fitoesteróis e os derivados polifenólicos (Moll, 2006).

Sabe-se que a dieta tem importante papel no desenvolvimento da doença coronariana, obesidade, diabetes tipo 2 e hipertensão. A dieta à base de vegetais e frutas contém substâncias antioxidantes e outras que contribuem para proteção e prevenção do desenvolvimento de doenças crônicas. Entre as diversas espécies de plantas há variação de até 1000 vezes na quantidade de substâncias antioxidantes encontradas nas plantas utilizadas na dieta. Algumas famílias de plantas apresentam maiores quantidades como Rosaceae, Empretraceae, Ericaceae, Grossulariaceae, Juglandaceae, Asteraceae, Punicaceae e Zingiberaceae (Halvorsen et al., 2002).

O Brasil tem grande biodiversidade apresentando também uma grande tradição no uso de plantas medicinais. Esta conjunção estabelece bom cenário para o desenvolvimento de pesquisas que visam a descoberta de novos fármacos a partir de espécies nativas (Leite, 2008).

A sistematização de dados referentes às espécies medicinais que possam subsidiar futuras pesquisas fitoquímicas e farmacológicas se faz necessária. Sendo assim, o objetivo deste trabalho foi fazer um levantamento a partir de livros publicados no Brasil entre 1998 e 2008, reunindo informações sobre plantas medicinas com atividade hipolipemiante, hipocolesterolêmica e/ou antiaterosclerótica, bem como verificar a existências de estudos científicos para as espécies nativas mais citadas. 


\section{MATERIAL E MÉTODO}

A pesquisa bibliográfica foi feita em 32 livros, de diversos autores, editados no Brasil entre 1998 e 2008: Almassy Júnior et al. (2005), Almeida (2000), Almeida et al. (1998), Alonso (2008), Balmé (2004), Barbosa Junior (2005), Botsaris (2002), Camargo (1998), Carvalho (2002), Correa et al. (2003), Di Stasi \& Hiruma-Lima (2002), Duniau (2003), Ferro (2008), Franco (2002), Franco (2003), Guião (2004), Lorenzi \& Matos (2002), Martins et al. (2000), Matos (1998), Matos (1999), Matos (2000), Ming (2006), Morgan (2003), Panizza (1999), Rodrigues et al. (2002), Rodrigues \& Carvalho (2001), Sartório et al. (2000), Schulz et al. (2002), Silva et al. (2008), Simões et al. (2004), Soares (2006) e Teske \& Trentini (2001). Foram utilizadas as palavras colesterol, hiperlipidemia, hipercolesterolemia e aterosclerose como termos de busca para esta pesquisa. A nomenclatura das espécies e das famílias foi atualizada de acordo com Souza \& Lorenzi (2005) e com o banco de dados do "The International Plant Names Index" (IPNI, 2008).

As espécies nativas citadas pelo menos duas vezes, nos livros consultados, foram submetidas à busca bibliográfica nas bases de dados Medline (2009), Lilacs (2009), Scielo (2009), Science Direct (2009) e Scirus (2009). Os termos utilizados para esta pesquisa foram o binômio científico com os termos, "hypolipidemic", "hypocholesterolemic" e "antiatherosclerotic".

\section{RESULTADO E DISCUSSÃO}

Os dados completos deste levantamento contêm citações de 85 espécies vegetais utilizadas no tratamento da hiperlipidemia, hipercolesterolemia e/ou aterosclerose, sendo que 54 são espécies exóticas e 31 são nativas (Tabela 1). As espécies citadas estão distribuídas em 53 famílias, sendo que as famílias com maior número de representantes citados foram Asteraceae, com 13 espécies e Fabaceae, com 9 espécies. Entretanto, os resultados encontrados nesta pesquisa, mostram que a maioria das espécies com maior número de citações não pertence às estas famílias, a exemplo de Allium sativum, que foi indicada em 18 livros e pertence à família Alliaceae. De acordo com Gottlieb \& Stefanello (1991), a Família Fabaceae aparece como uma das dez famílias mais citadas em levantamentos etnobotânicos publicados no Journal of Ethnopharmacology, o que pode ser explicado pelo fato de que estas famílias são constituídas de grande número de espécies se comparadas com outras do Reino Plantae (Judd et al., 1999).

TABELA 1. Plantas indicadas na literatura para o tratamento de hiperlipidemia, hipercolesterolemia e/ou aterosclerose. ( $\mathrm{N}=$ Nativa, E=Exótica).

\begin{tabular}{|c|c|c|c|c|c|}
\hline Família & Nome científico & Nome vulgar & Origem & $\begin{array}{c}\text { Parte } \\
\text { vegetal } \\
\text { utilizada }\end{array}$ & Referências \\
\hline Agavaceae & $\begin{array}{l}\text { Herreria salsaparrilha } \\
\text { Mart. }\end{array}$ & Salsaparrilha & $\mathrm{N}$ & Raiz & $\begin{array}{l}\text { Ferro (2008), Rodrigues \& Carvalho } \\
(2001)\end{array}$ \\
\hline Alismataceae & $\begin{array}{l}\text { Echinodorus } \\
\text { grandiflorus Mitch }\end{array}$ & $\begin{array}{l}\text { Chapéu de } \\
\text { couro }\end{array}$ & $N$ & Folha & $\begin{array}{l}\text { Almassy Júnior et al. (2005), Franco } \\
\text { (2002), Lorenzi \& Matos (2002), } \\
\text { Silva et al. (2008), Sartório et al. } \\
(2000)\end{array}$ \\
\hline \multirow[t]{2}{*}{ Alliaceae } & Allium cepa L. & Cebola & $E$ & Bulbo & $\begin{array}{l}\text { Almassy Júnior et al. (2005), Correa } \\
\text { et al. (2003), Ferro (2008), Morgan } \\
\text { (2003), Schulz et al.(2002), Silva et } \\
\text { al. (2008), Simões et al.(2004) }\end{array}$ \\
\hline & Allium sativum $\mathrm{L}$. & Alho & $E$ & Bulbo & $\begin{array}{l}\text { Almassy Júnior et al. (2005), Alonso } \\
\text { (2008), Barbosa Junior (2005), } \\
\text { Botsaris (2002), Correa et al. (2003), } \\
\text { Ferro (2008), Franco (2003), Franco } \\
\text { (2002), Guião (2004), Lorenzi \& } \\
\text { Matos (2002), Martins, et al. (2000), } \\
\text { Matos (1998), Matos (2000), Morgan } \\
\text { (2003), Panizza (1999), Schulz et al. } \\
\text { (2002), Silva et al. (2008), Teske \& } \\
\text { Trentini (2001) }\end{array}$ \\
\hline Amaranthaceae & $\begin{array}{l}\text { Pfaffia paniculata } \\
\text { (Mart.) Kuntze }\end{array}$ & $\begin{array}{l}\text { Ginseng- } \\
\text { brasileiro }\end{array}$ & $\mathrm{N}$ & Raíz & $\begin{array}{l}\text { Barbosa Junior (2005), Franco } \\
\text { (2002), Lorenzi \& Matos (2002) }\end{array}$ \\
\hline Anacardiaceae & $\begin{array}{l}\text { Anacardium } \\
\text { occidentale L. }\end{array}$ & Cajú & $\mathrm{N}$ & Amêndoa & Lorenzi \& Matos (2002) \\
\hline Apiaceae & $\begin{array}{l}\text { Angelica sinensis } \\
\text { (Oliv.) Diels. }\end{array}$ & $\begin{array}{l}\text { Angélica } \\
\text { chinesa }\end{array}$ & $E$ & Raiz & Botsaris (2002) \\
\hline Apiaceae & Daucus carota L. & Cenoura & $E$ & Fruto & $\begin{array}{l}\text { Almassy Júnior et al. (2005), } \\
\text { Botsaris (2002) }\end{array}$ \\
\hline Aquifoliaceae & $\begin{array}{l}\text { Ilex paranguariensis A. } \\
\text { St.-Hil. }\end{array}$ & Erva-mate & $\mathrm{N}$ & Folha & $\begin{array}{r}\text { Alonso (2008), Ferro (2008) } \\
\text { co }\end{array}$ \\
\hline
\end{tabular}


TABELA1. Plantas indicadas na literatura para o tratamento de hiperlipidemia, hipercolesterolemia e/ou aterosclerose. (N=Nativa, $\mathrm{E}=$ Exótica).

\begin{tabular}{|c|c|c|c|c|c|}
\hline Família & Nome científico & Nome vulgar & Origem & $\begin{array}{c}\text { Parte } \\
\text { vegetal } \\
\text { util izada }\end{array}$ & Referências \\
\hline Araliaceae & $\begin{array}{l}\text { Panax ginseng C. A. } \\
\text { Meyer }\end{array}$ & $\begin{array}{l}\text { Ginseng } \\
\text { coreano }\end{array}$ & $\mathrm{E}$ & Raiz & $\begin{array}{l}\text { Barbosa Junior (2005), Teske \& } \\
\text { Trentini (2001) }\end{array}$ \\
\hline \multirow[t]{13}{*}{ Asteraceae } & Arctium lappa L. & Bardana & $\mathrm{E}$ & $\begin{array}{c}\text { Planta } \\
\text { toda }\end{array}$ & Ferro (2008) \\
\hline & $\begin{array}{l}\text { Artemisia scoparia } \\
\text { Waldst. }\end{array}$ & Artemísia & E & $\begin{array}{l}\text { Parte } \\
\text { aérea }\end{array}$ & Simões et al. (2004) \\
\hline & $\begin{array}{l}\text { Baccharis trimera } \\
\text { (Less.)DC. }\end{array}$ & Carqueja & $\mathrm{N}$ & $\begin{array}{l}\text { Parte } \\
\text { aérea }\end{array}$ & $\begin{array}{l}\text { Ferro (2008), Franco (2002), Teske } \\
\text { \& Trentini (2001) }\end{array}$ \\
\hline & Calendula officinalis L. & Calêndula & $E$ & Flor & Ferro (2008) \\
\hline & $\begin{array}{l}\text { Chamomilla recutia (L.) } \\
\text { Rauschert }\end{array}$ & Camomila & $E$ & Flor & Silva et al. (2008) \\
\hline & Chicorium intybus L. & Chicória & $E$ & $\begin{array}{c}\text { Planta } \\
\text { toda }\end{array}$ & Ferro (2008) \\
\hline & Cynara scolymus L. & Alcachofra & E & Folha & $\begin{array}{l}\text { Alonso (2008), Correa et al. (2003), } \\
\text { Ferro (2008), Franco (2003) Franco } \\
\text { (2002), Guião (2004), Lorenzi \& } \\
\text { Matos (2002), Morgan (2003), } \\
\text { Panizza (1999), Sartório et al. } \\
\text { (2000), Silva et al. (2008), Soares } \\
\text { (2006), Teske \& Trentini (2001) }\end{array}$ \\
\hline & $\begin{array}{l}\text { Egletes viscosa (L.) } \\
\text { Less }\end{array}$ & Macela-da-terra & $\mathrm{N}$ & $\begin{array}{l}\text { Parte } \\
\text { aérea }\end{array}$ & Correa et al. (2003) \\
\hline & Lactuca sativa L. & Alface & E & Folha & Morgan (2003) \\
\hline & $\begin{array}{l}\text { Sylibum marianum }(\mathrm{L} .) \\
\text { Gaertner }\end{array}$ & Cardo-mariano & $E$ & Fruto seco & Ferro (2008) \\
\hline & $\begin{array}{l}\text { Taraxacum officinale } \\
\text { Weber }\end{array}$ & Dente de leão & $E$ & $\begin{array}{l}\text { Parte } \\
\text { aérea e } \\
\text { raíz }\end{array}$ & $\begin{array}{l}\text { Almassy Júnior et al. (2005), Ferro } \\
(2008), \text { Franco (2002), Martins, et al. } \\
(2000) \text {, Silva et al. (2008) }\end{array}$ \\
\hline & $\begin{array}{l}\text { Vernonia condensata } \\
\text { Baker }\end{array}$ & $\begin{array}{l}\text { Alumã, boldo- } \\
\text { chines }\end{array}$ & $E$ & Folha & $\begin{array}{l}\text { Barbosa Junior (2005), Ferro (2008), } \\
\text { Lorenzi \& Matos (2002), Panizza } \\
\text { (1999), Soares (2006) }\end{array}$ \\
\hline & $\begin{array}{l}\text { Polymnia sonchifolia } \\
\text { Griseb. }\end{array}$ & Yacon & E & Tubérculo & Ferro (2008) \\
\hline Betulaceae & Betula verrucosa Ehrh & Bétula & $E$ & Folha & Teske \& Trentini (2001) \\
\hline Bignoniaceae & $\begin{array}{l}\text { Tabebuia avellanedae } \\
\text { Lor. Griseb. }\end{array}$ & Ipê- roxo & $\mathrm{N}$ & $\begin{array}{l}\text { Casca e } \\
\text { lenho }\end{array}$ & $\begin{array}{l}\text { Barbosa Junior (2005), Ferro (2008), } \\
\text { Teske \& Trentini (2001) }\end{array}$ \\
\hline Bixaceae & Bixa orelana L. & Urucum & $\mathrm{N}$ & Semente & Ferro (2008) \\
\hline Boraginaceae & Cordia ecalyculata Vell & Porangaba & $\mathrm{N}$ & Folhas & Barbosa Junior (2005) \\
\hline Brassicaceae & Brassica oleraceae L. & Couve & $E$ & Folha & Ferro (2008) \\
\hline Capparac eae & Crateva tapia L. & Pau-d'alho & $\mathrm{N}$ & $\begin{array}{l}\text { Folha e } \\
\text { caule }\end{array}$ & Martins, et al. (2000) \\
\hline Chenopodiaceae & $\begin{array}{l}\text { Chenopodium } \\
\text { ambrosioides } \mathrm{L} .\end{array}$ & $\begin{array}{l}\text { Erva-de-santa- } \\
\text { maria }\end{array}$ & $\mathrm{N}$ & $\begin{array}{l}\text { Parte } \\
\text { aérea }\end{array}$ & Almassy Júnior et al. (2005) \\
\hline Curcubitaceae & $\begin{array}{l}\text { Mormodica charanthia } \\
\text { L. }\end{array}$ & $\begin{array}{l}\text { Melão-São- } \\
\text { Caetano }\end{array}$ & $E$ & $\begin{array}{l}\text { Folha e } \\
\text { fruto }\end{array}$ & Ferro (2008) \\
\hline Dioscoreaceae & Dioscorea villosa L. & $\begin{array}{l}\text { Inhame } \\
\text { mexic ano }\end{array}$ & $E$ & Tubérculo & Ferro (2008) \\
\hline Equisetaceae & Equisetum hiemale L. & Cavalinha & $E$ & $\begin{array}{l}\text { Parte } \\
\text { aérea }\end{array}$ & $\begin{array}{l}\text { Almassy Júnior et al. (2005), } \\
\text { Sartório et al. (2000), Teske \& } \\
\text { Trentini (2001) }\end{array}$ \\
\hline Eucommiaceae & $\begin{array}{l}\text { Eucommia ulmoides } \\
\text { Oliv. }\end{array}$ & - & $E$ & Casca & Botsaris (2002) \\
\hline Euphorbiaceae & Croton cajucara Benth & Sacaca & $\mathrm{N}$ & Folha & Lorenzi \& Matos (2002) \\
\hline \multirow[t]{3}{*}{ Fabaceae } & $\begin{array}{l}\text { Bauhinia cheilantha } \\
\text { (Bong.) Steu. }\end{array}$ & Pata-de-vaca & $\mathrm{N}$ & Folha & Lorenzi \& Matos (2002) \\
\hline & Bauhinia ungulata L. & $\begin{array}{l}\text { Mororó, unha- } \\
\text { de-vaca }\end{array}$ & $\mathrm{N}$ & $\begin{array}{l}\text { Folha e } \\
\text { casca }\end{array}$ & Matos (1998), Silva et al. (2008) \\
\hline & Bauhinia forficata Link. & Pata-de-vaca & $\mathrm{N}$ & Folha & $\begin{array}{l}\text { Correa et al. (2003), Guião (2004), } \\
\text { Matos (1998), Silva et al. (2008) }\end{array}$ \\
\hline
\end{tabular}


TABELA1. Plantas indicadas na literatura para o tratamento de hiperlipidemia, hipercolesterolemia e/ou aterosclerose. ( $\mathrm{N}=$ Nativa, $\mathrm{E}=$ Exótica). ... continuação

\begin{tabular}{|c|c|c|c|c|c|}
\hline Família & Nome científico & Nome vulgar & Origem & $\begin{array}{c}\text { Parte } \\
\text { vegetal } \\
\text { utilizada }\end{array}$ & Referências \\
\hline \multirow[t]{6}{*}{ Fabaceae } & $\begin{array}{l}\text { Dimorphandra mollis } \\
\text { Benth }\end{array}$ & Faveiro & $\mathrm{N}$ & $\begin{array}{l}\text { Fruto e } \\
\text { casca }\end{array}$ & Ferro (2008) \\
\hline & Glycine max $\mathrm{L}$. & Soja & $E$ & Semente & $\begin{array}{l}\text { Almassy Júnior et al. (2005), } \\
\text { Barbosa Junior (2005), Ferro (2008), } \\
\text { Schulz et al. (2002) }\end{array}$ \\
\hline & Glycyrrhiza glabra L. & $\begin{array}{l}\text { Alcaçuz } \\
\text { europeu }\end{array}$ & $E$ & Raíz & Botsaris (2002), Ferro (2008) \\
\hline & Medicago sativa $\mathrm{L}$. & Alfafa & $E$ & $\begin{array}{l}\text { Folha e } \\
\text { semente }\end{array}$ & $\begin{array}{l}\text { Ferro (2008), Lorenzi \& Matos } \\
(2002)\end{array}$ \\
\hline & Phaseolus vulgaris L. & Feijão & $\mathrm{E}$ & $\begin{array}{l}\text { Fruto sem } \\
\text { semente }\end{array}$ & Ferro $(2008)$ \\
\hline & $\begin{array}{l}\text { Pterodon emarginatus } \\
\text { Vogel }\end{array}$ & $\begin{array}{l}\text { Sucupira } \\
\text { branca }\end{array}$ & $\mathrm{N}$ & Semente & Ferro (2008) \\
\hline Fumariaceae & Fumaria officinalis $\mathrm{L}$. & Fumaria & $E$ & $\begin{array}{l}\text { Parte } \\
\text { aérea }\end{array}$ & $\begin{array}{l}\text { Almassy Júnior et al. (2005), Correa } \\
\text { et al. (2003), Morgan (2003), } \\
\text { Sartório et al. (2000) }\end{array}$ \\
\hline Ginkgoaceae & Ginkgo biloba L. & Ginkgo & $E$ & Folha & Ferro (2008), Franco (2002) \\
\hline Juglandaceae & Juglans cinerea L. & Nogueira & $\mathrm{E}$ & Folha & Ferro (2008) \\
\hline \multirow[t]{4}{*}{ Lamiaceae } & Leonorus sibiricus L. & Erva-macaé & $\mathrm{E}$ & $\begin{array}{l}\text { Parte } \\
\text { aérea }\end{array}$ & Ferro (2008) \\
\hline & Melissa officinalis $\mathrm{L}$. & Melissa & $E$ & $\begin{array}{l}\text { Parte } \\
\text { aérea }\end{array}$ & Silva et al. (2008) \\
\hline & Rosmarinus officinalis L. & Alecrim & $E$ & Folha & $\begin{array}{l}\text { Almassy Júnior et al. (2005), Franco } \\
\text { (2003), Silva et al. (2008) }\end{array}$ \\
\hline & $\begin{array}{l}\text { Plectranthus barbatus } \\
\text { Andrews }\end{array}$ & Falso-boldo & $E$ & Folha & Sartório et al. (2000) \\
\hline Lauraceae & Persea americana Mill. & Abacate & $E$ & Fruto & Franco (2003) \\
\hline Linaceae & Linum usitatissimum $\mathrm{L}$. & Linhaça & $E$ & Semente & Ferro (2008) \\
\hline Loranthaceae & Viscum album $\mathrm{L}$. & Visco & $\mathrm{E}$ & Folha & Correa et al. (2003) \\
\hline Lythaceae & $\begin{array}{l}\text { Cuphea carthagenensis } \\
\text { (Jacq) Mac }\end{array}$ & Sete-sangrias & $\mathrm{N}$ & $\begin{array}{l}\text { Planta } \\
\text { inteira }\end{array}$ & $\begin{array}{l}\text { Barbosa Junior (2005), Ferro (2008), } \\
\text { Lorenzi \& Matos (2002), Sartório et } \\
\text { al. (2000), Teske \& Trentini (2001) }\end{array}$ \\
\hline Malpighiaceae & Malphighia glaba L. & Acerola & $E$ & Fruto & Franco (2003) \\
\hline \multirow[t]{2}{*}{ Myrtaceae } & Eugenia uniflora L. & Pitangueira & $\mathrm{N}$ & $\begin{array}{l}\text { Folha e } \\
\text { fruto }\end{array}$ & Ferro (2008) \\
\hline & $\begin{array}{l}\text { Syzygium cumini (L.) } \\
\text { Skeels }\end{array}$ & Jambolão & $\mathrm{E}$ & Fruto & Almassy Júnior et al. (2005) \\
\hline Oleaceae & Olea europea L. & Azeitona & $E$ & Folha & Ferro (2008) \\
\hline Oxalidaceae & Averrhoa carambola L. & Carambola & $\mathrm{E}$ & Folha & Ferro (2008) \\
\hline Papaveraceae & Chelidonium majus L. & Celidônia & $\mathrm{E}$ & $\begin{array}{c}\text { Planta } \\
\text { toda }\end{array}$ & $\begin{array}{l}\text { Correa et al. (2003), Ferro (2008), } \\
\text { Lorenzi \& Matos (2002), Panizza } \\
\text { (1999) }\end{array}$ \\
\hline Piperaceae & $\begin{array}{l}\text { Peperomia pellucida } \\
\text { (L.) Kunth }\end{array}$ & Erva-jaboti & $\mathrm{N}$ & Folha & Lorenzi \& Matos (2002) \\
\hline \multirow[t]{2}{*}{ Poaceae } & Avena sativa $\mathrm{L}$. & Aveia & $E$ & Semente & $\begin{array}{l}\text { Almassy Júnior et al. (2005), Schulz } \\
\text { et al. (2002), Teske \& Trentini (2001) }\end{array}$ \\
\hline & Tricutum sativum Lank & Trigo & $E$ & Semente & Teske \& Trentini (2001) \\
\hline Polygonaceae & $\begin{array}{l}\text { Polygonum multiflorum } \\
\text { Thumb. }\end{array}$ & - & $\mathrm{N}$ & $\begin{array}{c}\text { Raiz } \\
\text { tuberosa } \\
\end{array}$ & Botsaris (2002) \\
\hline Punicaceae & Punica granatum L. & Romã & $E$ & Semente & Ferro (2008) \\
\hline \multirow[t]{2}{*}{ Rosaceae } & Crataegus oxycantha L. & $\begin{array}{l}\text { Espinheiro- } \\
\text { alvar }\end{array}$ & $E$ & Flor & Morgan (2003) \\
\hline & $\begin{array}{l}\text { Quillaja saponaria } \\
\text { Molina }\end{array}$ & Quilaia & $E$ & Casca & Simões et al. (2004) \\
\hline \multirow[t]{2}{*}{ Rubiaceae } & Coffea arabica L. & Café & $E$ & Semente & Lorenzi \& Matos (2002) \\
\hline & $\begin{array}{l}\text { Rudgea viburnoides } \\
\text { (Cham.) Bent. }\end{array}$ & $\begin{array}{l}\text { Congonha do } \\
\text { cerrado }\end{array}$ & $\mathrm{N}$ & Folha & Ferro (2008) \\
\hline
\end{tabular}


TABELA1. Plantas indicadas na literatura para o tratamento de hiperlipidemia, hipercolesterolemia e/ou aterosclerose. (N=Nativa, $\mathrm{E}=$ Exótica).

... continuação

\begin{tabular}{|c|c|c|c|c|c|}
\hline Família & Nome científico & Nome vulgar & Origem & $\begin{array}{c}\text { Parte } \\
\text { vegetal } \\
\text { utilizada }\end{array}$ & Referências \\
\hline \multirow[t]{2}{*}{ Rubiaceae } & $\begin{array}{l}\text { Rudgea viburnoides } \\
\text { (Cham.) Bent. }\end{array}$ & $\begin{array}{l}\text { Congonha do } \\
\text { cerrado }\end{array}$ & $\mathrm{N}$ & Folha & Ferro (2008) \\
\hline & $\begin{array}{l}\text { Uncaria tomentosa } \\
\text { (Willd.) DC. }\end{array}$ & Unha de gato & $\mathrm{N}$ & $\begin{array}{l}\text { Parte } \\
\text { aérea }\end{array}$ & Ferro (2008) \\
\hline Rutaceae & Citrus limonus L. & Limão & $\bar{E}$ & Fruto & $\begin{array}{l}\text { Almassy Júnior et al. (2005), Balmé } \\
\text { (2004), Correa et al. (2003), Ferro } \\
(2008)\end{array}$ \\
\hline Sapindaceae & Paullinia cupana Kunth & Guaraná & $\mathrm{N}$ & $\begin{array}{l}\text { Fruto e } \\
\text { semente }\end{array}$ & $\begin{array}{l}\text { Ferro (2008), Lorenzi \& Matos } \\
(2002)\end{array}$ \\
\hline Scrophulariaceae & Scoparia dulcis L. & Vassourinha & $\mathrm{N}$ & $\begin{array}{l}\text { Planta } \\
\text { toda }\end{array}$ & Matos (1998) \\
\hline Simaroubaceae & $\begin{array}{l}\text { Simaba ferruginea A. } \\
\text { St.Hill. }\end{array}$ & Calunga & $\mathrm{N}$ & Raíz & Ferro (2008) \\
\hline Smilacaceae & Smilax sp & Salsaparrilha & $\mathrm{N}$ & Raiz & $\begin{array}{l}\text { Barbosa Junior (2005), Panizza } \\
\text { (1999), Teske \& Trentini (2001) }\end{array}$ \\
\hline \multirow[t]{2}{*}{ Solanaceae } & $\begin{array}{l}\text { Licopersicon } \\
\text { esculentum Mill. }\end{array}$ & Tomate & $\bar{E}$ & Fruto & $\begin{array}{l}\text { Lorenzi \& Matos (2002), Matos } \\
(2000)\end{array}$ \\
\hline & Solanum melongena $\mathrm{L}$. & Beringela & $\mathrm{E}$ & Fruto & Ferro (2008), Panizza (1999) \\
\hline Theaceae & $\begin{array}{l}\text { Camellia sinensis (L.) } \\
\text { Kuntze }\end{array}$ & Chá-preto & $\bar{E}$ & Folha & $\begin{array}{l}\text { Guião (2004), Lorenzi \& Matos } \\
\text { (2002) }\end{array}$ \\
\hline Urticaceae & $\begin{array}{l}\text { Cecropia pachystachya } \\
\text { Trecul. }\end{array}$ & $\begin{array}{l}\text { Embaúba } \\
\text { branca }\end{array}$ & $\mathrm{N}$ & Folha & Almassy Júnior et al. (2005) \\
\hline Verbenaceae & $\begin{array}{l}\text { Stachytarpheta } \\
\text { caynnensis (Rich.)Vahl }\end{array}$ & Gervão & $\mathrm{N}$ & $\begin{array}{l}\text { Folha e } \\
\text { raíz }\end{array}$ & Ferro (2008) \\
\hline Violaceae & $\begin{array}{l}\text { Anchieta salutaris Saint } \\
\text { Bilair }\end{array}$ & Suma roxa & $\mathrm{N}$ & Raíz & Ferro (2008) \\
\hline Vitaceae & Vitis vinifera $\mathrm{L}$. & Uva & $E$ & Fruto & $\begin{array}{l}\text { Almassy Júnior et al. (2005), Alonso } \\
(2008)\end{array}$ \\
\hline \multirow[t]{2}{*}{ Zingiberaceae } & Curcuma longa L. & Açafroa & $E$ & Rizoma & $\begin{array}{l}\text { Botsaris (2002), Ferro (2008), } \\
\text { Lorenzi \& Matos (2002), Matos } \\
\text { (1998), Matos (1999), Matos (2000), } \\
\text { Panizza (1999), Sartório et al. } \\
\text { (2000), Silva et al. (2008) }\end{array}$ \\
\hline & $\begin{array}{l}\text { Curcuma zedoaria } \\
\text { (Christ.) Roscoe }\end{array}$ & Zedoária & $E$ & Rizoma & $\begin{array}{l}\text { Alonso (2008), Barbosa Junior } \\
\text { (2005), Lorenzi \& Matos (2002), } \\
\text { Panizza (1999), Teske \& Trentini } \\
\text { (2001) }\end{array}$ \\
\hline
\end{tabular}

As espécies mais citadas nos livros consultados foram Allium sativum (Alliaceae) com 18 citações, Cynara scolymus (Asteraceae) com 13 citações, Curcuma longa (Zingiberaceae) com 9 citações, Allium cepa (Alliaceae) com 7 citações, Echinodorus grandiflorus (Alismataceae), Taraxacum officinale (Asteraceae), Vernonia condensata (Asteraceae), Cuphea carthagenensis (Lythraceae) e Curcuma zedoaria (Zingiberaceae) com 5 citações (Tabela 1).

O alho (Allium sativum) é conhecido na Europa como planta medicinal curativa desde a idade média. Os efeitos antiaterosclerótico e de redução de lipídeos atribuídos ao alho eram desconhecidos na medicina antiga e medieval, pois a aterosclerose não era uma doença importante até a época industrial. Os efeitos do alho têm sido extensivamente documentados por pesquisas clínicas e farmacológicas. As maiorias dos estudos farmacológicos foram feitos em modelos animais e tratam de efeitos antiaterogênico, redutor de gordura, anti-hipertensivo, efeito inibitório sobre a síntese de colesterol, propriedades vasodilatadoras e antioxidante. Estes efeitos são atribuídos aos compostos contendo enxofre, a aliina, ácido alisufênico e alicina (Schulz et al., 2002; Alonso, 2008). O mecanismo molecular de ação destes compostos é baseado na inibição da enzima chave da biossíntese do colesterol, a HMGCoA redutase (Gebhardt \& Beck, 1996).

As espécies nativas têm sido largamente empregadas pela população, cujo conhecimento acerca do uso medicinal foi desenvolvido inicialmente por comunidades indígenas e caboclas do país (Leite, 2008). A Tabela 2 mostra as espécies nativas citadas, destacando-se a Cuphea carthagenensis, 
TABELA2. Espécies nativas indicadas para o tratamento de hiperlipidemia, hipercolesterolemia e/ou aterosclerose.

\begin{tabular}{|c|c|c|}
\hline Nome científico / Família & Termos encontrados nos livros & $\begin{array}{c}\text { Número } \\
\text { de } \\
\text { citações }\end{array}$ \\
\hline Anacardium occidentale L. (Anacardiaceae) & Redução do colesterol e triglicerideos & 1 \\
\hline Anchieta salutaris Saint Bilair (Violaceae) & Aterosclerose & 1 \\
\hline Baccharis trimera (Less.)D.C. (Asteraceae) & Dislipidemias, redução do colesterol & 3 \\
\hline Bauhinia cheilantha (Bong.) Steud. (Fabaceae) & Redução do colesterol e triglicerideos & 1 \\
\hline Bauhinia forficata Link. (Fabaceae) & Hipercolesterolemia & 4 \\
\hline Bauhinia ungulata L. (Fabaceae) & Hipolipemiante & 2 \\
\hline Bixa orelana L. (Bixaceae) & Hipocolesterolemiante & 1 \\
\hline Cecropia pachystachya Trecul. (Urticaceae) & Aterosclerose & 1 \\
\hline Chenopodium ambrosioides L. (Chenopodiaceae) & Aterosclerose & 1 \\
\hline Cordia ecalyculata Vell ( Boraginaceae) & Redução do colesterol & 1 \\
\hline Crataeva tapia L. (Cappraceae) & Redução do colesterol & 1 \\
\hline Croton cajucara Benth (Euphorbiaceae) & Baixar o colesterol & 1 \\
\hline Cuphea carthagenensis (Jacq) Macbr (Lythaceae) & Aterosclerose, hipocolesterolêmia & 5 \\
\hline Dimorphandra mollis Benth (Fabaceae) & Prevenção da ateroscleorose & 1 \\
\hline Echinodorus grandiflorus Mitch (Alismataceae) & Aterosclerose, hipocolesterolêmia & 5 \\
\hline Egletes viscosa (L.) Less (Asteraceae) & Hipercolesterolemia & 1 \\
\hline Eugenia uniflora L. (Myrtaceae) & Dislipidemias & 1 \\
\hline Herreria salsaparrilha Mart. (Agavaceae) & Redução do colesterol & 2 \\
\hline Ilex paranguariensis A. St.-Hil. (Aquifoliaceae) & Lipolítica, redutor de colesterol & 2 \\
\hline Paullinia cupana Kunth (Sapindaceae) & Aterosclerose & 2 \\
\hline Peperomia pellucida (L.) Kunth (Piperaceae) & Aterosclerose & 1 \\
\hline Pfaffia paniculata (Mart.) Kuntze (Amaranthaceae) & Redução do colesterol & 3 \\
\hline Polygonum multiflorum Thumb. (Polygonaceae) & Aterosclerose, hipocolesterolêmia & 1 \\
\hline Pterodon emarginatus Vogel (Fabaceae) & Dislipidemias & 1 \\
\hline Rudgea viburnoides L. (Cham.) Benth. (Rubiaceae) & Dislipidemias & 1 \\
\hline Scoparia dulcis L. (Scrophulariaceae) & Redução do colesterol e triglicerídeos & 1 \\
\hline Simaba ferruginea A. St.Hill. (Simaroubaceae) & Dislipidemias & 1 \\
\hline Smilax spp (Smilacaceae) & Redução do colesterol & 3 \\
\hline Stachytarpheta caynnensis (Rich.) Vahl (Verbenaceae) & Dislipidemias & 1 \\
\hline Tabebuia avellanedae Lor. et Griseb. (Bignoniaceae) & Aterosclerose & 3 \\
\hline Uncaria tomentosa (Willd.) DC. (Rubiaceae) & Aterosclerose & 1 \\
\hline
\end{tabular}

Echinodorus grandiflorus, Bauhinia forficata, Tabebuia avellanedae, Smilax sp, Pfaffia paniculata, Baccharis trimera, llex paraguariensis, Paullinia cupana e Herreria salsaparrilha.

Brito \& Brito (1993) apontam diversos estudos químicos e/ou farmacológicos realizados com espécies da flora nativa, ressaltando as potencialidades de utilização de várias delas, bem como a necessidade de maiores estudos com estas espécies, principalmente devido a grande biodiversidade do Brasil.

Apesar do Brasil possuir cerca de $20 \%$ de todas as plantas e microorganismos existentes no planeta (Calixto, 2005), este levantamento revela que ainda são poucas as pesquisas com as espécies nativas e as respectivas atividades hipolipidêmica e antiaterosclerótica. Este é o caso de Herreria salsaparrilha, espécie nativa citada em 2 livros pesquisados, para a qual não foi encontrado nenhum estudo químico ou farmacológico.

Para a espécie E. grandiflorus, planta nativa conhecida como chapéu-de-couro, foi feita a análise fitoquímica de partes aéreas que revelou a presença de 17 componentes, dentre eles os de maior concentração foram o fitol e sesquiterpenos (Pimenta et al., 2006). Tibiriçá et al. (2007) demonstraram, em experimento in vitro, que o extrato aquoso $(0,1$ a 10 $\mathrm{mg}$ ) desta planta apresenta potente efeito 
vasodilatador que possivelmente, se deu em parte pela via de ativação do óxido nítrico-GMPc. A via do óxido nítrico-GMPc, desempenha um importante papel no relaxamento vascular de músculo liso, na adesão de plaquetas e leucócitos e na permeabilidade endotelial. A redução na produção de óxido nítrico pelas células do endotélio vascular está associada com a disfunção endotelial em injúrias às quais podem ser importantes fatores em doenças cardiovasculares especialmente no desenvolvimento da aterosclerose e hipertensão (Busse \& Fleming, 1996). Então o desenvolvimento de substâncias com possíveis propriedades vasodilatadoras que atuam restaurando os níveis de óxido nítrico-GMPc no sistema vascular podem ser de grande valor para o tratamento de doenças cardiovasculares.

Entre as espécies nativas mais citadas, $C$. carthagenensis é mais estudada sob o ponto de vista da atividade hipolipidêmica. Em estudo realizado na região sul do Brasil, C. carthagenensis foi utilizada em estudos pré-clinicos que indicaram possível papel no controle da hiperlipidemia, condição muitas vezes associada à obesidade (Dickel et al., 2007). Esta espécie também foi investigada para atividade antioxidante utilizando sistema de peroxidação em lipídeos e ficou demonstrado que os extratos hidroalcoolico, butanólico e de acetato de etila causaram inibição da peroxidação de lipídeos em homogenatos de fígado de ratos. Os autores sugeriram que extratos de folhas de $C$. carthagenensis são fontes ricas em compostos fenólicos, com atividade antioxidante in vitro e poderiam ter efeitos benéficos em doenças cardiovasculares (Schuldt et al., 2004). Análises bioquímicas demonstraram a redução do colesterol no plasma de ratos tratados, em longo prazo, com extrato a $2 \%$ desta planta (Biavatti et al., 2004). Outro estudo com extrato butanólico de $C$. carthagenensis verificou, em artéria aorta de ratos, a capacidade de relaxamento do endotélio, o que poderia ser indicação de uso desta planta para doenças cardiovasculares (Schuldt et al., 2000).

Bauhinia forficata, outra planta nativa citada neste levantamento, tem a maioria das pesquisas direcionadas ao estudo da sua indicação como antidiabético (Silva et al., 2002; Jorge et al., 2004; Lino et al., 2004; Pinheiro et al., 2006; Khalil et al., 2008), não tendo sido encontrado nenhum relato científico que possa comprovar a indicação desta espécie para o tratamento de hiperlipidemia. Para a espécie $B$. ungulata os trabalhos encontrados resultam de estudos químicos onde foram identificadas a presença de flavonoides e alcalóides nesta espécie (Salatino et al.,1999;Maia Neto, 2008).

Baccharis trimera, espécie muito utilizada pela população brasileira para perda de peso (Dickel et al., 2007), tem sido investigada quanto a atividade antidiabética (Oliveira et al., 2005; Frode \& Medeiros, 2008).

Tabebuia avellanedae, conhecida como ipê roxo, tem sido muito estudada pelas propriedades antitumorais, antiinflamatórias e antineoplásicas (Miranda et al., 2001; Kung et al., 2008; Queiroz et al., 2008). Byeon et al. (2008) estudaram os efeitos antiinflamatórios de extrato aquoso desta planta in vitroe in vivo, e constataram que a ação farmacológica de $T$. avellanedae se deve a inibição da resposta inflamatória mediada por macrófagos pela supressão de prostaglandina 2 (substância pró-inflamatória). Desta forma, sugerem que o extrato das folhas desta espécie pode ser desenvolvido para o tratamento de doenças inflamatórias como artrite e aterosclerose. Estudos preliminares com extrato de folhas de Tabebuia heptaphylla, identificaram a presença de flavonóides e demonstraram inibir fortemente a peroxidação lipídica induzida por $\mathrm{H}_{2} \mathrm{O}_{2}$ e $\mathrm{FeSO}_{4}$. Este efeito pode explicar a indicação do uso na prevenção de aterosclerose, relatadas na medicina popular (Budni et al., 2007).

Estudos com Smilax sp têm sido conduzidos principalmente para demonstrar a atividade antioxidante de extratos de folhas e de raízes desta planta medicinal conhecida como salsaparrilha (Demo et al., 1998; Tripathi et al., 2001; Cox et al., 2005; Hee et al., 2006; Ivanova et al., 2006; Ozsoy et al., 2008). Os efeitos antioxidantes desta espécie são atribuídos aos flavonóides identificados, que podem ser indicadores do controle de qualidade desta planta como a isoramnetina, camferol e quercetina (Rugna et al., 1999; Rugna et al., 2004; Rugna et al., 2007; Shao et al., 2007; Rugna et al., 2008; Xu et al., 2008).

A espécie Pfaffia paniculata é conhecida como o ginseng brasileiro pelas raízes tuberosas parecidas com as de Panax ginseng (Araliaceae). A literatura científica não apresenta nenhum relato sobre a atividade hipocolesterolêmica desta espécie, sendo que as atividades farmacológicas deste gênero versam basicamente sobre a droga na forma de pó da raiz, com extrato bruto ou semi-purificado (Vigo et al., 2004). Um dos principais efeitos da P. paniculata se deve a atividade antitumoral do ácido fáfico e fafosídeos (Nishimoto, 1984; Pinello et al., 2006).

Estudos já comprovaram que plantas com alto teor de flavonóides são utilizadas na prevenção e tratamento de hiperlipidemia, hipercolesterolemia e aterosclerose (IV Diretrizes da SBC, 2007). Porém, o uso destes vegetais e as formulações devem ser vistos com cautela. Estudo comparativo feito entre o extrato aquoso de llex paraguariensis e o produto comercial chamado "Erva Mate", que demonstrou que o extrato aquoso apresentou maior atividade da peroxidase do que o produto comercial da mesma planta. Maior atividade desta enzima indica maior

Rev. Bras. PI. Med., Botucatu, v.13, n.1, p.98-109, 2011. 
capacidade antioxidante que está relacionada ao maior conteúdo de compostos fenólicos (Anesini et al., 2006). l. paraguariensis contém muitos compostos bioativos como ácidos fenólicos que parecem ser responsáveis pela atividade antioxidante das infusões de chá verde tanto in vitro quanto in vivo (Gugliucci, 1996; Schinella et al., 2000; Bracesco et al., 2003; Bastos et al., 2006).

Os trabalhos sobre Paullinia cupana(guaraná) tratam de revisões sobre possíveis efeitos adversos do uso desta planta como suplemento alimentar para perda de peso (Andersen \& Fogh, 2001; Pittler, 2005) e sobre efeitos adversos cardiovasculares como taquicardia, atribuídos à presença de cafeína na planta (Baghkhani \& Jafari, 2002).

Entre as espécies nativas citadas em apenas um livro (Tabela 2), podemos ressaltar Croton cajucara, que foi alvo de revisão sobre as atividades biológicas da trans-desidrocrotonina, obtida desta planta (Costa et al., 2007) e relata atividade hipolipidemica encontrada em diversos estudos (Silva et al., 2001a; Silva et al., 2001b; Silva et al., 2001c; Bighetti et al., 2004; Silva et al., 2005).

As plantas com atividade antioxidante comprovadas podem ser direcionadas para estudos farmacológicos e clínicos para hiperlipidemia, hipercolesterolemia e aterosclerose tornando-se alvos potenciais para desenvolvimento de novas drogas ou como terapia adjuvante no tratamento e prevenção de doenças.

Ainda são poucos os dados científicos relacionados à atividade hipocolesterolêmica de espécies brasileiras, indicando a necessidade de estudos que possam garantir a segurança e a eficácia do uso destas espécies.

\section{REFERÊNCIA}

ALMASSY JÚNIOR, A.A. et al. Folhas de chá: plantas medicinais na terapêutica humana. Viçosa: Editora Universidade Federal de Viçosa, 2005. 233p.

ALMEIDA, M.Z. Plantas medicinais. Salvador: Editora Universidade Federal da Bahia, 2000. 192p.

ALMEIDA, S.M. et al. Cerrado: espécies vegetais úteis. Planaltina: EMBRAPA-CPAC, 1998. 464p.

ALONSO, J. Fitomedicina: curso para profissionais da área de saúde. São Paulo: Editora Pharmabooks, 2008. $195 p$.

ANDERSEN, T.; FOGH, J. Weight loss and delayed gastric emptying following a South American herbal preparation in overweight patients. Journal of Human Nutrition and Dietetics, v.14, p.243-50, 2001.

ANESINI, C. et al. Peroxidase-like activity of /lex paraguariensis. Food Chemistry, v.97, p.459-64, 2006. ANILA, L.; VIJAYALAKSHMI, N.R. Flanonoids from Emblica officinalis and Mangifera indica - effectiveness for dyslipidemia. Journal of Ethnopharmacology, v.79, p.817, 2002.
BAGHKHANI, L.; JAFARI, M. Cardiovascular adverse reactions associated with guarana: Is there a causal effect? Journal of Herbal Pharmacotherapy, v.2, p.57-61, 2002. BALMÉ, F. Plantas medicinais. São Paulo: Editora Hemus, 2004. 398p.

BARBOSA JÚNIOR, A. Plantas medicinais: descubra o que os vegetais podem fazer pela sua saúde. São Paulo: Universo dos Livros, 2005. 126p.

BASTOS, D.H.M. et al. Essential oil and antioxidant activity of green mate and mate tea (/lex paraguariensis) infusions. Journal of Food Composition and Analysis, v.19, p.538-43, 2006.

BIAVATTI, M.W. et al. Preliminary studies on Campomanesia xanthocarpa (Berg.) and Cuphea carthagenensis (Jacq.) J.F. Macbr. aqueous extract: weight control and biochemical parameters. Journal of Ethnopharmacology, v.93, p.385-9, 2004.

BIGHETTI, E.J.B. et al. Chronic treatment with bark infusion from Croton cajucara lowers plasma triglyceride levels in genetic hyperlipidemic mice. Canadian Journal of Physiology and Pharmacology, v.82, p.387-92, 2004. BORRADAILE, N.M. et al. Inhibition of hepatocyte apoB secretion by naringenin: enhanced rapid intracellular degradation independent of reduced microsomal cholesteryl esters. Journal of Lipid Research, v.43, p.1544-54, 2002.

BORRADAILE, N.M. et al. Hepatocyte ApoB-containing lipoprotein secretion is decreased by the grapefruit flavonoid, naringenin, via inhibition of MTP-mediated microssomal triglyceride accumulation. Biochemistry, v.42, p.1283-91, 2003.

BOTSARIS, A.S. Fitoterapia chinesa e plantas brasileiras. 2.ed. São Paulo: Editora Ícone, 2002. 550p. BRACESCO, N. et al. Antioxidant activity of a botanical extract preparation of Ilex paraguariensis: prevention of DNA doublestrand breaks in Saccharomyces cerevisiae and human low-density lipoprotein oxidation. Journal of Alternative and Complementary Medicine, v.9, p.37987, 2003.

BRITO, A.R.M.S.; BRITO, A.A.S. Forty years of Brazilian medicinal plant research. Journal of Ethnopharmacology, v.39, p.53-67, 1993.

BRUSQ, J.M. et al. Inhibition of lipid synthesis through activation of AMK- Kinase: an additional mechanism for hypolipidemic effects of Beberine. Journal of Lipid Research, v.47, p.1281-8, 2006.

BUDNI, P. et al. Preliminary studies of the antioxidant activity of adult and young leaf extract hydroetanclic of Tabebuia heptaphylla (Vell.). Latin American Journal of Pharmacy, v.26, p.394-8, 2007.

BUSSE, R.; FLEMING, I. Endothelial dysfunction in atherosclerosis. Journal of Vascular Research, v.33, p.181-94, 1996.

BYEON, S.E. et al. In vitro and in vivo anti-inflammatory effects of taheebo, a water extract from the inner bark of Tabebuia avellanedae. Journal of Ethnopharmacology, v.119, p.145-52, 2008.

CALIXTO, J.B. Twenty-five years of research on medicinal plants in Latin América: A personal view. Journal of Ethnopharmacology, v.100, p.131-4, 2005.

CAMARGO, M.T.A. Plantas medicinais e de rituais afrobrasileiros II: Estudo etnofarmacobotanico. São Paulo: Icone Editora, 1998. 232p. 
CARVALHO, A.F. Ervas e temperos: cultivo, processamento e receitas. Viçosa: Aprenda Fácil, 2002. 296p.

COON, J.S.T.; ERNST, E. Herbs for serum cholesterol reduction: a systematic review. The Journal of Family Practice, v.52, p.468-78, 2003.

CORREAA, A.D. et al. Plantas medicinais: do cultivo à terapêutica. 6.ed. Petrópolis: Editora Vozes, 2003. 248p. COSTA, M.P. et al. Uma revisão das atividades biológicas da trans-desidrocrotonina, um produto natural obtido de Croton cajucara. Revista Brasileira de Farmacognosia, v.17, p.275-86, 2007.

COTRAN, R.S. et al. Robbins: patologia estrutural e funcional. Rio de Janeiro: Editora Guanabara-Koogan, 2000. 1213p.

COX, S.D. et al. Antioxidant activity in Australian native sarsaparilla (Smilax glyciphylla). Journal of Ethnopharmacology, v.101, p.162-8, 2005.

DEMO, A. et al. Nutrient antioxidants in some herbs and Mediterranean plant leaves. Food Research International, v.31, p.351-4, 1998.

DI STASI, L.C.; HIRUMA-LIMA, C.A. Plantas medicinais na Amazônia e Mata Atlântica. 2.ed. São Paulo:Editora UNESP, 2002. 604p.

DICKEL, M.L. et al. Plants popularly used for loosing weight purposes in Porto Alegre, South Brazil. Journal of Ethnopharmacology, v.109, p.60-71, 2007.

DUNIAU, M.C.M. Plantas medicinais: da magia a ciência. Rio de Janeiro: Editora Brasport, 2003.150p.

FERRO, D. Fitoterapia: conceitos clínicos. São Paulo: Editora Atheneu, 2008. 502p.

FRANCO, L.L. As sensacionais $\mathbf{5 0}$ plantas medicinais: campeãs do poder curativo. 5.ed. Curitiba: Naturista, 2002. 241p.

FRANCO, L.L. Doenças tratadas com plantas medicinais. 2.ed. Petrópolis: Editora Vozes, 2003. 144p. FRÖDE, T.S.; MEDEIROS, Y.S. Animal models to test drugs with potential antidiabetic activity. Journal of Ethnopharmacology, v.115, p.173-83, 2008.

GEBHARDT, R.; BECK, H. Differencial Inibitory effects of Garlic-derived organosulfur compounds on cholesterol Biosynthesis in Primary rat hepatocyte Cultures. Lipids, v.31, p.1269-76, 1996.

GOTTLIEB, O.R.E.; STEFANELLO, M.E.A. Comparative ethnopharmacology: a racional method for the search of bioactive compounds in plants. Anais da Academia Brasileira de Ciências, v.63, p.23-31, 1991.

GUGLIUCCI, A. antioxidant effects of Ilex paraguariensis: induction of decreased oxidability of human LDL in vivo. Biochemical and Biophysical Research Communications, v.224, p.338-44, 1996.

GUIÃO, M. et al. Plantas medicinais: cultivo, utilidades e comercialização. Belo Horizonte: EMATER-MG/ Prorenda rural-MG, IEF-MG. Doces Matas, 2004. 192p.

HALVORSEN, B.L. et al. A systematic screening of total antioxidants in dietary plants. Journal of Nutrition, v.132, p.461-71, 2002.

HEE, S.S. et al. Antioxidant activity of extracts from Smilax china root. Journal of the Korean Society of Food Science and Nutrition, v.35, p.1133-8, 2006.

SOCIEDADE BRASILEIRA DE CARDIOLOGIA. IV Diretrizes Brasileiras Sobre Dislipidemias e Diretriz de Prevenção da Aterosclerose do Departamento de Aterosclerose da Sociedade Brasileira de Cardiologia.
Arquivos Brasileiros de Cardiologia, v.88, supl. 1, p.119, 2007.

IPNI. The International Plant Names Index. 2008. Disponível em: <http://www.ipni.org>. Acesso em: 01 out. 2008.

IVANOVA, A. et al. Antioxidant properties of Smilax excelsa. Rivista Italiana delle Sostanze Grasse, v.83, p.124-8, 2006. JEON, S.M. et al. Antihypercholesterolemic property of naringin alters plasma and tissue lipids, cholesterolregulating enzymes, fecal sterol and tissue morphology in rabbits. Clinical Nutrition, v.23, p.1025-34, 2004.

JORGE, A.P. et al. Insulinomimetic effects of kaempferitrin on glycaemia and on 14C-glucose uptake in rat soleus muscle. Chemico-Biological Interactions, v.49, p.89-96, 2004.

JUDD, W.S.; CAMPBELL, C.S.; KELLOGG, P.F. Plant systematics: a phylogenetic approach. Massachusetts: Sinauer Associates, 1999. cap. 8, p.161-419.

JUNG, U.J. et al. Effect of citrus flavonoids on lipid metabolism and glucose-regulating enzyme mRNA levels in type-2 diabetic mice. International Journal of Biochemistry and Cell Biology, v.38, p.1134-45, 2006.

KHALIL, N.M. et al. Free radical scavenging profile and myeloperoxidase inhibition of extracts from antidiabetic plants: Bauhinia forficata and Cissus. Biological Research, v.41, p.165-71, 2008.

KONG, W.J. et al. Berberine is a novel cholesterol-lowering drug working through a unique mechanism distinct from statins. Nature Medicine, v.10, p.1344-51, 2004.

KUNG, H.N. et al. In vitro and in vivo wound healingpromoting activities of beta-lapachone. American Journal of Physiology, v.295, p.931-43, 2008.

LEITE, J.P.V. Fitoterapia: bases científicas e tecnológicas. São Paulo: Atheneu, 2008. 328p.

LILACS. Biblioteca Virtual em Saúde. 2009. Disponível em: <http://www.bireme.br> Acesso em: 20 jan. 2009.

LINO, C.S. et al. Antidiabetic activity of Bauhinia forficata extracts in alloxan-diabetic rats. Biological and Pharmaceutical Bulletin, v.27, p.125-7, 2004.

LORENZI, H.; MATOS, F.J.A. Plantas medicinais do Brasil: nativas e exóticas. Nova Odessa: Plantarum, 2002. 512p.

MAIA NETO, M. et al. Flavonoids and alkaloids from leaves of Bauhinia ungulata L. Biochemical Systematics and Ecology, v.36, p.227-9, 2008.

MARINANGELI, C.P.F. et al. Plant sterols combined with exercise for the treatment of hypercholesterolemia: overview of independent. Journal of Nutritional Biochemistry, v.17, p.217-24, 2006.

MARTINS, E.R. et al. Plantas medicinais. Viçosa: Editora da Universidade Federal de Viçosa, 2000. 220p.

MATOS, F.J.A. Farmácias vivas. 3.ed. Fortaleza: EUFC, 1998. 218p.

MATOS, F.J.A. Plantas da medicina popular do nordeste: propriedades atribuídas e confirmadas. Fortaleza: Imprensa Universitária, 1999. 80p.

MATOS, F.J.A. Plantas medicinais: guia de seleção e emprego de plantas usadas em fitoterapia no nordeste brasileiro. 2.ed. Fortaleza: Imprensa Universitária, 2000. $346 p$.

MEDLINE. PubMed - National Library of Medicine. 2009. Disponível em: <http://bases.bireme.br> and http://www. ncbi.nlm.nih.gov/books/bv.fcgi. Acesso em: 20 fev. 2009. 
MING, L.C. Plantas medicinais na reserva extrativista Chico Mendes: uma visão etnobotânica. São Paulo: Editora UNESP, 2006. 160p.

MIRANDA, F.G.G. et al. Antinociceptive and antiedematogenic properties and acute toxicity of Tabebuia avellanedae Lor. ex Griseb. inner bark aqueous extract. BMC Pharmacology, v.1, p.1-6, 2001. MOLL, M.C.N. Antihiperlipemiantes de origen vegetal. Revista de Fitoterapia, v.6, p.11-26, 2006.

MORGAN, R. Enciclopédia das ervas e plantas medicinais. 9.ed. São Paulo: Editora Hemus, 2003. 555p. NISHIMOTO, N. et al. Pfaffosides and nortreterpenoid saponins from Pffafia paniculata. Phytochemistry, v.23, p.139-42, 1984.

OLIVEIRA, A.C.P. et al. Effect of the extracts and fractions of Baccharis trimera and Syzygium cumini on glycaemia of diabetic and non-diabetic mice. Journal of Ethnopharmacology, v.102, p.465-9, 2005.

OZSOY, N. et al. Antioxidant activity of Smilax excelsa L. leaf extracts. Food Chemistry, v.110, p.571-83, 2008.

PANIZZA, S. Plantas que curam: cheiro de mato. 20.ed. São Paulo: Editora Ibrasa, 1999. 279p.

PIMENTA, D.S. et al. Essential oil from two populations of Equinodorus grandiflorus Micheli. Anais da Academia Brasileira de Ciências, v.78, p.623-8, 2006.

PINELLO, K.C. et al. Efects of Pfaffia paniculata (Brasilian Ginseng) extract on macrophage activity. Life Sciences, v.78, p.1287-92, 2006.

PINHEIRO, T.S.D.B. et al. Comparative assessment of kaempferitrin from medicinal extracts of Bauhinia forficata Link. Journal of Pharmaceutical and Biomedical Analysis, v.41, p.431-6, 2006.

PITTLER, M.H. Adverse events of herbal food supplements for body weight reduction: systematic review. Obesity Reviews, v.6, p.93-111, 2005.

QUEIROZ, M.L.S. et al. Comparative studies of the effects of Tabebuia avellanedae bark extract and betalapachone on the hematopoietic response of tumourbearing mice. Journal of Ethnopharmacology, v.117, p.228-35, 2008.

RODRIGUES, A.G. et al. Plantas medicinais e aromáticas: etnoecologia e etnofarmacologia. Viçosa: Editora da UFV, 2002. 320p.

RODRIGUES, V.E.G.; CARVALHO, D.A. Plantas medicinais no domínio dos Cerrados. Lavras: Editora UFLA, 2001. 180p.

RUGNA, A.Z. et al. Progress in studies on flavonols from Smilax campestris Griseb. - Smilacaceae. Acta Horticulturae, v.501, p.191-4, 1999.

RUGNA, A.Z. et al. Estudio comparativo de flavonoides en los organos aereos de Smilax campestris Griseb. Smilacaceae. Acta Farmacêutica Bonaerense, v.23, p.379-82, 2004.

RUGNA, A.Z. et al. Production rythm of polyphenols from Smilax campestris Griseb. (Smilacaceae). Boletin Latinoamericano y del Caribe de Plantas Medicinales y Aromaticas, v.6, p.297-8, 2007.

RUGNA, A.Z. et al. Variation in leaves polyphenol content in Smilax campestris Griseb. - Smilacaceae - according to their development. Latin American Journal of Pharmacy, v.27, p.247-9, 2008.

SALATINO, A. et al. Foliar flavonoids of nine species of Bauhinia. Revista Brasileira de Botânica, v.22, p.17-20,
1999.

SARTÓRIO, M.L. et al. Cultivo orgânico de plantas Medicinais. Viçosa: Editora Aprenda Fácil, 2000. 260p. SCHINELLA, G.R. et al. Antioxidant effects of an aqueous extract of Ilex paraguariensis. Biochemical and Biophysical Research Communications, v.269, p.35760, 2000.

SCHULDT, E.Z. et al. Butanolic fraction from Cuphea carthagenensis Jacq. mcbride relaxes rat thoracic aorta through endothelium-dependent and endotheliumindependent mechanisms. Journal of Cardiovascular Pharmacology, v.35, p.234-9, 2000.

SCHULDT, E.Z. et al. Comparative study of radical scavenger activities of crude extract and fractions from Cuphea carthagenensis leaves. Phytomedicine, v.11, p.523-9, 2004.

SCHULZ, V. et al. Fitoterapia racional: um guia de fitoterapia para as ciências da saúde. Barueri: Ed Manole, 2002. 386p.

SCIELO. Scientific Eletronic Library Online. Disponível em: <http://www.bireme.br>. Acesso em: 20 jan. 2009.

SCIENCE DIRECT. Disponível em: <http://www. sciencedirect. com>. Acesso em: 14 jan. 2009.

SCIRUS. Disponível em: <http://www.scirus.com>. Acesso em: 16 jan. 2009.

SHAO, B. et al. Simultaneous determination of six major stilbenes and flavonoids in Smilax china by high performance liquid chromatography. Journal of Pharmaceutical and Biomedical Analysis, v.44, p.73742, 2007.

SILVA, F. et al. Folhas de chá: remédios caseiros e comercialização de plantas medicinais, aromáticas e condimentares. Viçosa: Editora Universidade Federal de Viçosa, 2008. 140p.

SILVA, F.R.M.B. et al. Acute effect of Bauhinia forficata on serum glucose levels in normal and alloxan-induced diabetic rats. Journal of Ethnopharmacology, v.83, p.337, 2002.

SILVA, R.M. et al. Effect of trans-dehydrocrotonin, a 19nor-clerodane diterpene from Croton cajucara on experimental hypertriglyceridaemia and hypercholesterolaemia induced by triton WR1339 (tyloxapol) in mice. Planta Medica, v.67, p.763-5, 2001a.

SILVA, R.M. et al. Blood glucose- and triglyceride-lowering effect of trans-dehydrocrotonin, a diterpene from Croton cajucara Benth., in rats. Diabetes, Obesity and Metabolism, v.3, p.452-6, 2001 b.

SILVA, R.M. et al. The lipid-lowering effect of transdehydrocrotonin, a clerodane diterpene from Croton cajucara Benth. in mice fed on high-fat diet. Journal of Pharmacy and Pharmacology, v.53, p.535-9, 2001c.

SILVA, R.M. et al. Cardiovascular effects of transdehydrocrotonin, a diterpene from Croton cajucara in rats. Vascular Pharmacology, v.43, p.11-8, 2005.

SIMÕES, C.M.O. et al. Farmacognosia: da planta ao medicamento. 5.ed. Porto Alegre/Florianópolis: Editora da UFRS/UFSC, 2004. 1102p.

SINGI, G. et al. Efeitos agudos dos extratos hidroalcólicos do alho (Allium sativum L.) e do capim-limão (Cymbopogon citratus (DC.) Stapf) sobre a pressão arterial média de ratos anestesiados. Revista Brasileira de Farmacognosia, v.15, n.2, p.94-7, 2005.

SOARES, C.A. A cura que vem dos chás. Petrópolis: 
Editora Vozes, 2006. 264p.

SOUZA, V.C.; LORENZI, H. Botânica sistemática. Nova Odessa: Plantarum, 2005. 640p.

TESKE, M.; TRENTINI, A.M.M. Herbarium: compêndio de fitoterapia. 4.ed. Curitiba: Herbarium, 2001. 317p.

TIBIRIÇÁ, E. et al. Pharmacological mechanisms involved in the vasodilator effects of extracts from Echinodorus grandiflorus. Journal of Ethnopharmacology, v.111, p.50-5, 2007.

TRIPATHI, Y.B. et al. Antioxidant property of Smilax china Linn. Indian Journal of Experimental Biology, v.39, p.1176-9, 2001.

VIGO, C.L.S. et al. Caracterização farmacognóstica comparativa de Pfaffia glomerata (Spreng.) Pederson e Hebanthe paniculata Martius-Amaranthacceae. Revista Brasileira de Plantas Medicinais, v.6, p.7-19, 2004.

WANG, H.X.; NG, T.B. Natural products with hypoglycemic, hypotensive, hypocholesterolemic, antiatherosclerotic and antithrombotic activities. Life Sciences, v.65, p.266377, 1999.

WHITMAN, S.C. et al. Nobiletin, a citrus flavonoid isolated from tangerines, seletively inhibits class A scavenger receptor-mediated metabolism of acetylated LDL by mouse macrophages. Atherosclerosis, v.178, p.25-32, 2005.

$\mathrm{XU}, \mathrm{W}$. et al. Kaempferol-7-O-beta-D-glucoside (KG) isolated from Smilax china L. rhizome induces $\mathrm{G}(2) / \mathrm{M}$ phase arrest and apoptosis on HeLa cells in a p53independent manner. Câncer Letters, v.264, p.229-40, 2008.

YUNES, R.A. et al. Fármacos e fitoterápicos: a necessidade do desenvolvimento da indústria de fitoterápicos e fitofármacos no Brasil. Química Nova, v.24, p.147-52, 2001. 